\title{
Impact of Atrial fibrillation diagnosis or Carotid endarterectomy requirement on outcome in patients from the Triple Antiplatelets for Reducing Dependency after Ischaemic Stroke (TARDIS) trial
}

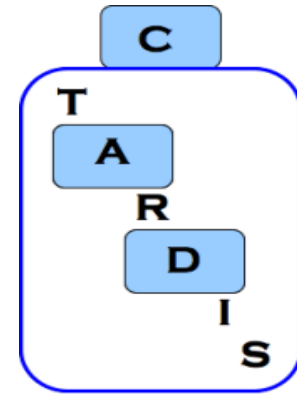

\author{
Lisa J Woodhouse, Jason P Appleton, Katie Flaherty, Rob Dineen, Nikola Sprigg, Philip M Bath \\ for the TARDIS investigators \\ Stroke, Division of Clinical Neuroscience, University of Nottingham, City Hospital Campus, \\ Nottingham, UK \\ Website: www.tardistrial.org Email: tardis@nottingham.ac.uk
}

\section{BACKGROUND}

- Carotid stenosis and atrial fibrillation (AF) are common causes of ischaemic stroke (IS) and transient ischaemic attack (TIA), and require carotid endarterectomy (CEA) and anticoagulation respectively to reduce recurrence risk.

- We assessed whether these interventions improve outcome using data from the TARDIS trial.

\section{METHOD}

- TARDIS assessed one month of intensive antiplatelet therapy versus guideline in 3096 patients with acute non-cardioembolic IS or TIA.

- Information regarding CEA requirement, AF diagnosis, recurrence and functional outcome was assessed centrally blinded to treatment assignment.

- Data are unadjusted odds ratio (OR) or mean difference (MD) with 95\% confidence interval (CI) relative to no $A F / C E A$.

\section{RESULTS}

Of the 3096, 3070 (99.2\%) patients had data regarding AF and CEA: $118(3.8 \%)$ patients were diagnosed with $\mathrm{AF}$ and $84(2.7 \%)$ required a CEA.

- In comparison with others, AF and CEA patients were older (4.2 and 2.4 years respectively) and more likely to have a history of ischaemic heart disease (IHD) than the rest (Table 1).

- AF patients were also more likely to have been enrolled with stroke rather than a TIA (Table 1).

Table 1. Baseline Characteristics

\begin{tabular}{lcccc} 
& AF & No AF & CEA & No CEA \\
\hline $\mathrm{N}$ & 118 & 2952 & 84 & 2986 \\
Age & $73(9.5)$ & $68.8(10.1)$ & $71.3(10.2)$ & $68.9(10.1)$ \\
Male \% & $66(55.9)$ & $1868(63.3)$ & $63(75.0)$ & $1871(62.7)$ \\
History IHD \% & $23(19.5)$ & $378(12.8)$ & $20(23.8)$ & $381(12.8)$ \\
Stroke \% & $100(84.7)$ & $2026(68.6)$ & $66(78.6)$ & $2060(69.0)$ \\
\hline
\end{tabular}

\section{RESULTS (Continued)}

- By day 90, AF patients were more likely to have a stroke/TIA recurrence (Table 2, OR $2.31,95 \% \mathrm{CI}$ 1.30-4.10; 7 before diagnosis, 9 afterwards).

- They also had a shift to more dependency (modified Rankin Scale, OR 1.50, 95\% Cl 1.07-2.10, Figure 1) and disability (Barthel Index, MD $-6.5,95 \% \mathrm{Cl}-10.0$ to -3.1 ).

- Outcomes of CEA patients were not significantly different from patients who did not require CEA.

Table 2. Day 90 outcomes for patients with and without AF

\begin{tabular}{lcccc} 
& AF & No AF & OR/MD (95\% Cl) & P \\
\hline Recurrence (\%) & $15(12.7)$ & $183(6.2)$ & $2.31(1.30,4.10)$ & 0.0044 \\
- Stroke (\%) & $13(11.0)$ & $105(3.6)$ & $3.45(1.84,6.45)$ & $<0.001$ \\
- TIA (\%) & $2(1.7)$ & $86(2.9)$ & $0.64(0.15,2.65)$ & 0.53 \\
mRS [/6] & $2[1,3]$ & $1[1,2]$ & $1.50(1.07,2.10)$ & 0.018 \\
Barthel Index & $83.3(32.2)$ & $93.2(18.4)$ & $-6.5(-10.0,-3.1)$ & $<0.001$ \\
EQ-5D (HUS) & $0.6(0.4)$ & $0.8(0.3)$ & $-0.1(-0.1,0.0)$ & 0.014 \\
\hline
\end{tabular}

Figure 1. Distribution of $\mathrm{mRS}$ in patients with and without AF

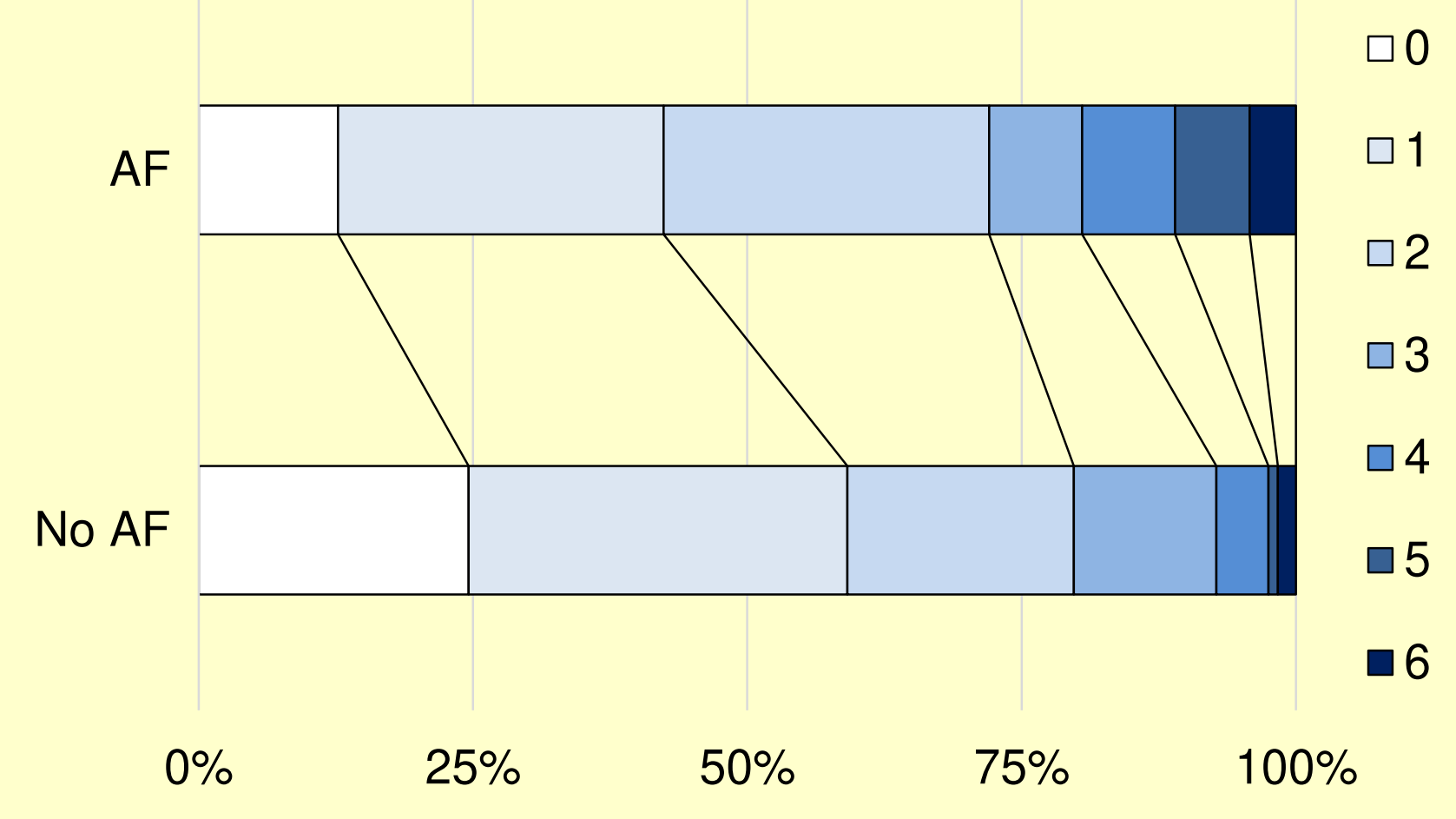

\section{CONCLUSIONS}

- Recurrence was more common in patients with AF than in other TARDIS patients; patients with AF also had worse functional outcomes.

- There was no difference in recurrence or functional outcomes between patients who did and did not require CEA. 